\title{
Armazenamento refrigerado de inflorescências cortadas de Oncidium varicosum 'Samurai'
}

\author{
Cold storage of cut inflorescences of Oncidium varicosum 'Samurai'
}

\author{
Claudia Fabrino Machado Mattiuz ${ }^{\text {I* }}$ Teresinha de Jesus Deléo Rodrigues ${ }^{\text {II }}$ \\ Ben-Hur Mattiuz ${ }^{I}$ Júlia de Pietro $^{\mathrm{I}}$ Ramilo Nogueira Martins ${ }^{1}$
}

\section{RESUMO}

A eficiência da baixa temperatura no prolongamento da vida de vaso de várias flores de corte tem sido bastante estudada. No entanto, o efeito da temperatura depende da duração do armazenamento e da espécie floral. $O$ experimento foi realizado objetivando avaliar a longevidade e as alterações metabólicas ocorridas durante o armazenamento refrigerado de inflorescências cortadas de Oncidium varicosum 'Samurai', armazenadas em diferentes temperaturas $5,10 \mathrm{e}$ $20^{\circ} \mathrm{C}$ ). Foram avaliados os seguintes parâmetros: conteúdo relativo de água, carboidratos solúveis e açúcares redutores, conteúdo de carotenóides, coloração e longevidade. Os resultados indicaram diferenças nos parâmetros avaliados nas três temperaturas de armazenamento. As flores de Oncidium varicosum 'Samurai', armazenadas a $5^{\circ} \mathrm{C}$, apresentaram melhor manutenção da qualidade do que nas outras temperaturas, o que foi evidenciado pela menor redução do conteúdo relativo de água, melhor manutenção do conteúdo de carotenóides, dos carboidratos solúveis e açúcares redutores, coloração e maior longevidade.

Palavras-chave: orquideas, chuva-de-ouro, flor-de-annita, pós-colheita, temperatura.

\section{ABSTRACT}

The effectiveness of low temperatures on extending vase life of several cut flowers has been largely investigated. However, the temperature effect depends on the length of the storage period and of the floral species. The experiment was accomplished to evaluate the longevity and the metabolic changes which occurred during the cold storage of cut inflorescences of Oncidium varicosum 'Samurai' kept under different temperatures $\left(5,10\right.$ and $\left.20^{\circ} \mathrm{C}\right)$. The following parameters were analyzed: relative water content (RWC), soluble carbohydrates, reducing sugars, carotenoids contents, color and longevity. The results indicated differences among the measured parameters at the three storage temperatures. The inflorescences of Oncidium varicosum 'Samurai' stored at $5^{\circ} \mathrm{C}$ presented better maintenance of flowers quality than at the other temperatures. It was evidenced by the lower reduction of $R W C$, better maintenance of carotenoids, soluble carbohydrates and reducing sugars. The flowers kept at $5{ }^{\circ} \mathrm{C}$ also presented maintenance of coloration and greater longevity.

Key words: orchids, golden shower, Annita's flower, postharvest, temperature.

\section{INTRODUÇÃO}

Oncidium varicosum é uma orquídea autóctone da Mata Atlântica no estado do Rio de Janeiro, como descrito por MILLER et al. (1996). Recentemente, sua importância comercial tem aumentado bastante, em decorrência do seu potencial de utilização como flor de corte. A espécie Oncidium varicosum caracteriza-se por seus pseudobulbos ovais ou elípticos comprimidos lateralmente, labelo largo tetralobado, ornado de elevações verruciformes na base e pelas inúmeras flores amarelas que compõem sua inflorescência ereta e ramificada (CORREA, 1984).

As flores necessitam de condições adequadas para manter seu frescor, coloração e

'Departamento de Tecnologia, Faculdade de Ciências Agrárias e Veterinárias (FCAV), Universidade Estadual Paulista (UNESP), Via de Acesso Prof. Paulo Donato Castellane, s/n. 14884-900, Jaboticabal, SP, Brasil. E-mail: cmattiuz@gmail.com. *Autor para correspondência.

"Departamento de Biologia Aplicada à Agropecuária, FCAV, UNESP, Jaboticabal, SP, Brasil. 
longevidade após a colheita. A refrigeração é considerada um aspecto de grande relevância para a comercialização de flores, pois possibilita a manutenção da qualidade, o armazenamento e o transporte a longas distâncias. $\mathrm{O}$ armazenamento em temperatura baixa diminui a transpiração, reduz a produção de etileno, diminui a respiração, retarda a degradação das reservas de açúcares e, por isso, prolonga a durabilidade das flores (NOWAK et al., 1991).

Estudos realizados em espécies de orquídeas tropicais, do gênero Phalaenopsis e Dendrobium, revelaram que temperaturas baixas têm efeito promotor nos teores endógenos de citocininas. A esse hormônio têm sido atribuído efeito retardador da senescência e maior longevidade floral (KERBAUY, 2004).

Muitas espécies de origem tropical e subtropical e algumas de origem em clima temperado podem apresentar distúrbios fisiológicos causados pelo frio, quando expostas a temperaturas entre $0 \mathrm{e}$ $15^{\circ} \mathrm{C}$ (KAYS, 1991). Segundo HALEVY \& MAYAK (1981) flores tropicais como orquídea, antúrio, ave-doparaíso, alpina e helicônia devem ser armazenadas em temperaturas superiores a $10^{\circ} \mathrm{C}$. WILLS et al. (1998) relata resultados benéficos do armazenamento refrigerado, em temperaturas entre 5 e $15^{\circ} \mathrm{C}$, de orquídeas tropicais como Vanda, Dendrobium e Cattleya.

Durante o armazenamento, observa-se que a progressão rápida dos sintomas de senescência pode indicar que a temperatura e/ou a duração do armazenamento não são adequadas para a espécie (KETSA\& THAMPITAKORN, 1995).

Foi observado em orquídeas Dendrobium 'Pompidour' que o pré-resfriamento a $10^{\circ} \mathrm{C}(85-95 \%$ de umidade relativa), por 60 minutos, reduziu a produção de etileno e da atividade do ácido 1-aminociclopropano 1-carboxílico (ACC) (KETSA et al., 2005).

O objetivo do presente trabalho foi avaliar o efeito do armazenamento refrigerado na conservação pós-colheita de inflorescências cortadas de Oncidium varicosum 'Samurai'.

\section{MATERIAL E MÉTODOS}

Inflorescências de Oncidium varicosum 'Samurai' foram colhidas pela manhã, com 30 por cento das flores em ponto de abertura floral, em área de produção comercial do município de Atibaia-SP. Em seguida, cada haste foi inserida em um tubo plástico vedado (com dimensões de $6,0 \times 2,0 \mathrm{~cm}$ ), contendo $10 \mathrm{~mL}$ de solução de ácido cítrico (pH, 4,2) e embalada em filme plástico de polietileno microperfurado transparente. O transporte das flores para o laboratório de Tecnologia dos Produtos Agropecuários da FCAVUNESP, Jaboticabal, SP, foi realizado em veículo refrigerado. O tempo compreendido entre a colheita e a chegada ao laboratório foi de quatro horas.

As flores foram armazenadas em câmaras de refrigeração de fluxo contínuo, com medidores de temperatura e de umidade relativa. No interior de cada câmara, foram colocados dois recipientes de $500 \mathrm{~mL}$ contendo água, para a manutenção da umidade relativa entre $90-95 \%$.

O delineamento experimental empregado foi o inteiramente casualizado, em um bifatorial: armazenamento refrigerado $\left(5,10\right.$ e $\left.20^{\circ} \mathrm{C}\right)$ e dias de avaliação $(0,5,10$ e 15 dias). Cada tratamento foi composto por três repetições, com cinco inflorescências, para cada dia de avaliação.

A avaliação da coloração das inflorescências foi feita utilizando-se colorímetro MINOLTA CR 400, determinando-se os valores de $\mathrm{L}(100=$ branco; $0=$ preto), $a^{*}($ positivo $=$ vermelho; negativo $=$ verde $), b^{*}$ (positivo=amarelo; negativo=azul). O ângulo Hue $\left(\mathrm{h}^{\circ}\right)$, ou de cor, e a cromaticidade foram calculados com o método descrito em MINOLTA CORP (1994).

O conteúdo relativo de água foi obtido pela coleta de dez flores inteiras, em cada repetição. As flores foram pesadas e imersas em água destilada, para hidratação, por um período de quatro horas. Após esse período, as flores foram novamente pesadas e levadas para estufa de secagem, a $70^{\circ} \mathrm{C}$ (KRAMER, 1983).

Os carboidratos solúveis das flores de Oncidium foram extraídos em etanol, utilizando-se o método fenol-sulfúrico (DUBOIS, et al., 1956; ROBYT \& WHITE, 1987) e os açúcares redutores com o método descrito em PAULL \& CHANTRACHIT (2001).

Para a determinação do conteúdo de carotenóides totais, em cada tratamento foram pesadas amostras de $1 \mathrm{~g}$ de pétalas por repetição. Cada amostra foi macerada em acetona a $80 \%(\mathrm{v} / \mathrm{v})$ na presença de $\mathrm{CaCO}_{3}$. A leitura das absorbâncias foi realizada em espectrofotômetro (Beckman - DU - 640), nos comprimentos de onda de 480,645 e $663 \mathrm{~nm}$ e os carotenóides totais foram calculados de acordo com HENDRY \& PRICE (1993).

A vida de vaso foi avaliada diariamente e foi considerada encerrada quando $50 \%$ ou mais flores de uma inflorescência haviam perdido sua qualidade ornamental. Os dias de vida de vaso (DVV) de cada tratamento foram contabilizados e esse valor correspondeu à longevidade média da inflorescência.

Os dados obtidos foram submetidos à análise de variância e as médias foram comparadas pelo teste de diferença mínima significativa em teste de 
comparações múltiplas. Assim, as diferenças entre dois tratamentos, quando maior que a soma de dois desvios padrões, foram consideradas significativas em nível de $5 \%$ de probabilidade (SHAMAILA et al., 1992).

\section{RESULTADOS E DISCUSSÃO}

Na figura 1, observa-se que inicialmente ocorreu um pequeno aumento do conteúdo relativo de água das inflorescências após cinco dias de armazenamento refrigerado $\left(5\right.$ e $\left.10^{\circ} \mathrm{C}\right)$, o que pode ter ocorrido devido à maior absorção inicial, promovida pelo condicionamento das hastes florais na solução de ácido cítrico. A inclusão de ácidos orgânicos, como o ácido cítrico, tem como principal função o abaixamento do $\mathrm{pH}$ das soluções. MAROUSKY (1971) afirmou que o $\mathrm{pH}$ ácido em soluções conservantes resulta em aumento na durabilidade de flores cortadas. Esse efeito do $\mathrm{pH}$ foi explicado por ROGERS (1973), ao relatar que soluções ácidas podem inibir a ação de enzimas endógenas, responsáveis pelo bloqueio da haste, ou impedir o desenvolvimento de microrganismos.

Aos dez dias de refrigeração, pôde-se observar que ocorreram diferenças significativas entre os tratamentos, com destaque para o tratamento a $5^{\circ} \mathrm{C}$ que manteve o maior teor $(88,38 \%$ ) de água nas flores (Figura 1). A maior turgescência dessas inflorescências indica que houve um efeito benéfico conjunto entre a absorção da solução conservante e a temperatura de armazenamento. O equilíbrio entre a absorção de água, seu transporte e a transpiração permite um balanço hídrico que favorece a manutenção da turgescência, necessária para o desenvolvimento dos botões florais até a abertura da flor e também para a continuidade da atividade metabólica normal da flor cortada. Aos 15 dias de armazenamento, as inflorescências armazenadas nas temperaturas de 10 e $20^{\circ} \mathrm{C}$ já haviam perdido sua qualidade decorativa. A exposição à temperatura inadequada durante longos períodos é considerada a principal causa de descarte de flores, nos locais de comercialização. No caso de órgãos florais, há grande perda de água por transpiração, em decorrência de sua grande superfície de exposição (KAYS, 1991; WILLS et al., 1998).

Na figura 2, encontram-se os resultados da avaliação do conteúdo de carotenóides das flores de Oncidium e observa-se que, aos dez dias de refrigeração, ocorreram diferenças significativas entre os tratamentos. A temperatura de $5^{\circ} \mathrm{C}$ se destacou significativamente das demais e as inflorescências apresentaram maior concentração de carotenóides no décimo dia $\left(292,72 \mu \mathrm{mol} \mathrm{g}\right.$ de matéria fresca $\left.{ }^{-1}\right)$ e no décimo quinto dia $\left(288,21 \mu \mathrm{mol} \mathrm{g}\right.$ de matéria fresca $\left.^{-1}\right)$, em relação ao início e ao quinto dia. O teor de carotenóides, expresso em função da massa fresca, pode ter maior valor devido à perda de água e podendo também ter ocorrido síntese durante o período. Considerando-se que o desbotamento é um fator prejudicial à qualidade decorativa de flores, o emprego de uma temperatura adequada durante o período de

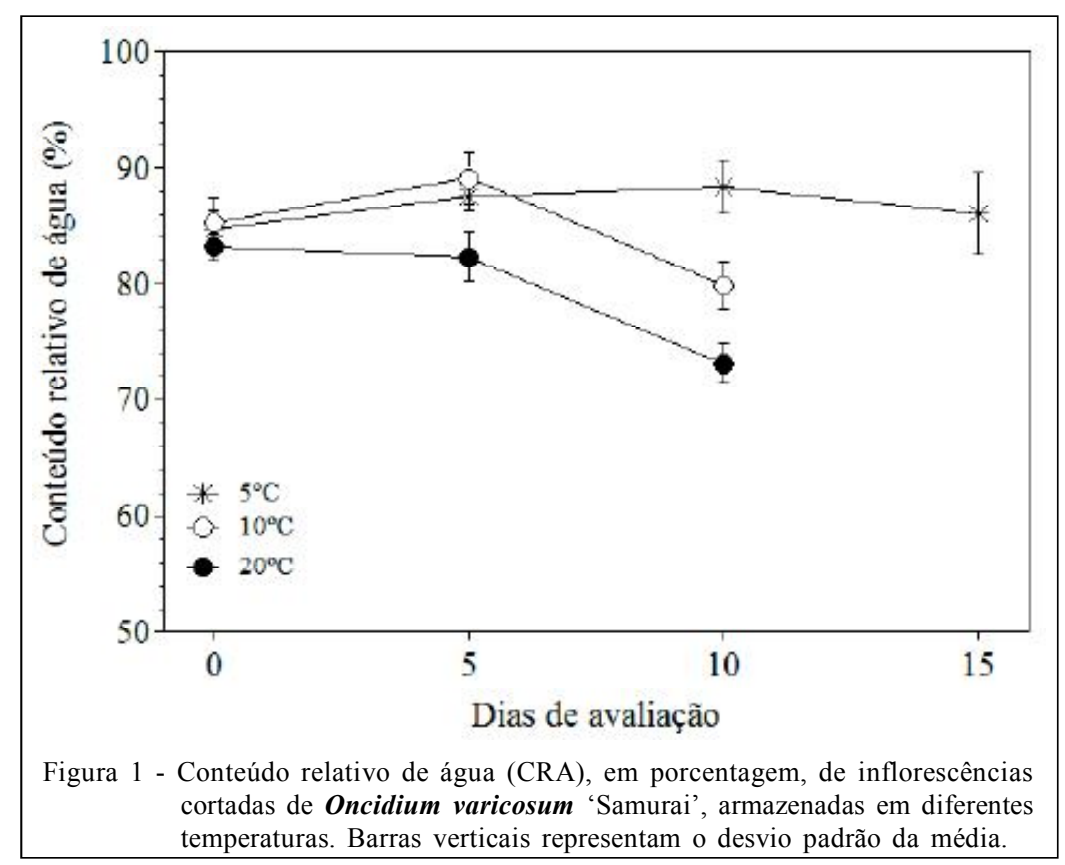

Ciência Rural, v.40, n.11, nov, 2010. 


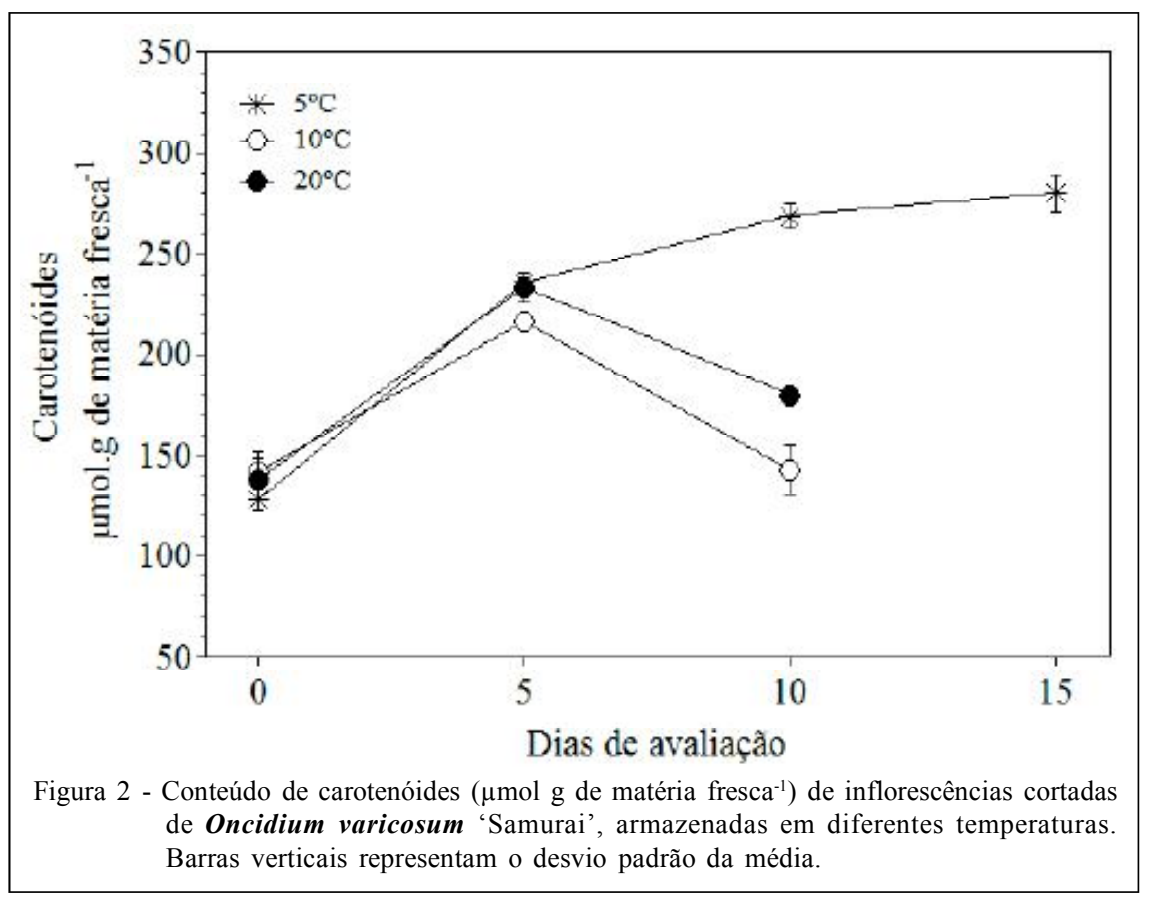

armazenamento pode prevenir a degradação precoce dos pigmentos nas pétalas florais, durante a comercialização. Segundo DEMMING-ADAMS (1996), a concentração de carotenóides é variável nas diferentes espécies florais e também pode variar de acordo com a progressão dos sintomas de senescência.

Os resultados obtidos da avaliação de coloração encontram-se na figura 3, na qual se observa que houve uma diminuição na luminosidade das inflorescências ao longo do período de avaliação, para todos os tratamentos. Sabendo que a luminosidade (MINOLTACORP., 1994) é representada por uma escala de zero (preto) a cem (branco), observa-se uma tendência de maior claridade nas inflorescências armazenadas a $5^{\circ} \mathrm{C}$. Para o ângulo de cor, ocorreu, desde o início, uma tendência de redução, em todos os tratamentos. No entanto, até o $10^{\circ}$ dia de vida de vaso não foi observada diferença significativa entre as temperaturas. O uso da refrigeração por tempo prolongado pode causar alterações nos padrões de coloração e, mesmo que inicialmente estas mudanças não sejam perceptíveis ao olho humano, certamente interferirão na estabilidade da cor, como demonstrado por meio da degradação dos pigmentos carotenóides (Figura 2).

A cromaticidade também apresentou resultados semelhantes, porém, aos dez dias de armazenamento, o tratamento a $20^{\circ} \mathrm{C}$ apresentou a menor média, diferindo significativamente dos demais, e indicou uma menor vivacidade da cor. Esse resultado é um indicativo de que as temperaturas superiores a $5^{\circ} \mathrm{C}$ afetam a qualidade da cor das flores de Oncidium varicosum 'Samurai'.

A sensibilidade de uma planta ou parte dela ao dano pelo frio pode variar em função da espécie, da cultivar e do tempo de exposição. Os sintomas desse distúrbio em espécies tropicais caracterizam-se pelo extravasamento hídrico dos tecidos, murchamento, perda de coloração e surgimento de lesões necróticas nas pétalas e sépalas (NOWAK \& RUDNICKI, 1990). Nas flores de Oncidium, mantidas a 5 e $10^{\circ} \mathrm{C}$, não foram observadas lesões necróticas.

$\mathrm{Na}$ figura 4, encontram-se os resultados de carboidratos solúveis e de açúcares redutores, ao longo do período de armazenamento das inflorescências de Oncidium 'Samurai'. No quinto dia de refrigeração, observou-se um aumento inicial do conteúdo de carboidratos solúveis e de açúcares redutores, que possivelmente foi aumentos nos teores de açúcares quando as plantas foram submetidas a baixas temperaturas (KERBAUY, 2004).

Aos dez dias, ocorreram diferenças significativas entre os tratamentos, com superioridade para o tratamento a $5^{\circ} \mathrm{C}$, cujas flores apresentaram 17,74 e $21,52 \mathrm{~g}$ de glicose $100 \mathrm{~g}^{-1}$, enquanto que a $10^{\circ} \mathrm{C}$ foram 8,04 e $10,11 \mathrm{~g}$ de glicose $100 \mathrm{~g}^{-1}$ e a $20^{\circ} \mathrm{C}$ apresentaram 4,31 e $7,73 \mathrm{~g}$ de glicose $100 \mathrm{~g}^{-1}$, para carboidratos solúveis e açúcares redutores, respectivamente. De 


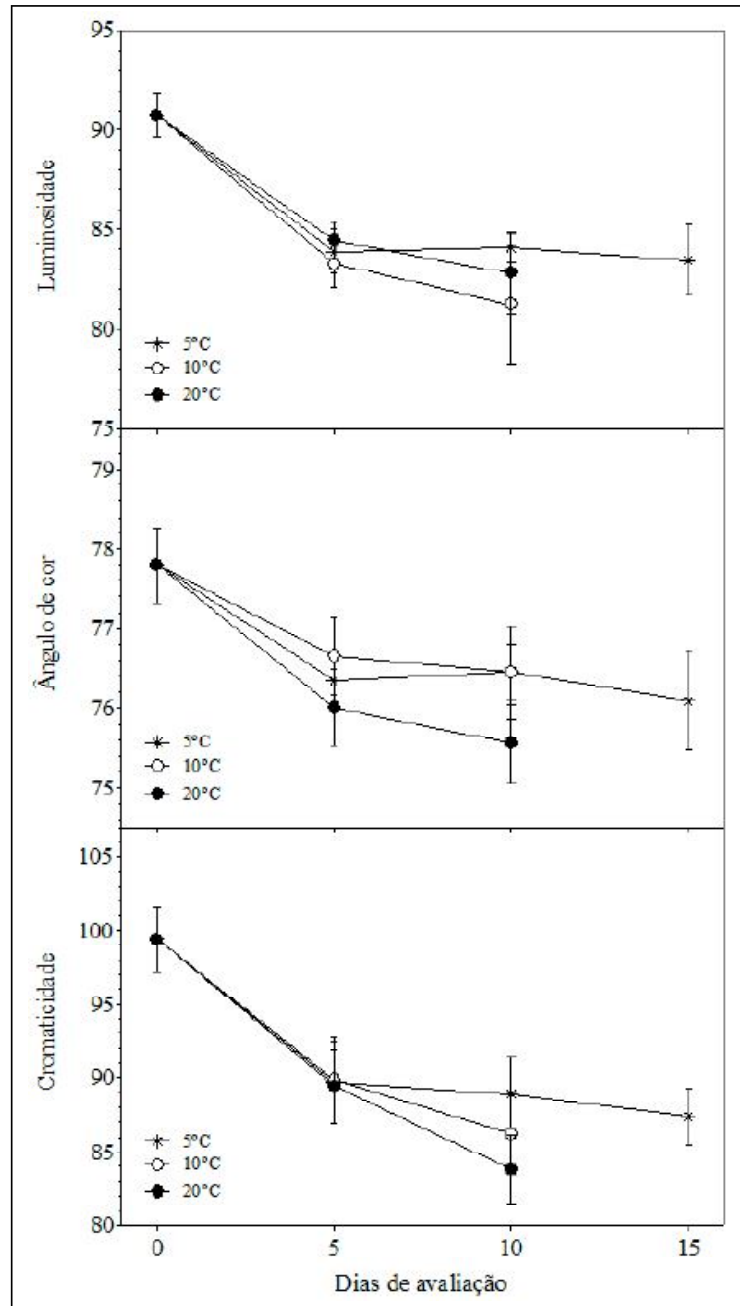

Figura 3 - Luminosidade, ângulo de cor e cromaticidade de inflorescências cortadas de Oncidium varicosum 'Samurai', armazenadas em diferentes temperaturas. Barras verticais representam o desvio padrão da média.

acordo com KERBAUY (2004), na maioria dos tecidos vegetais, um aumento de $10^{\circ} \mathrm{C}$, na faixa $5-25^{\circ} \mathrm{C}$, dobra a taxa respiratória devido ao aumento da atividade enzimática. Abaixo de $5^{\circ} \mathrm{C}$, há uma diminuição drástica da taxa respiratória, enquanto que ao redor de $30^{\circ} \mathrm{C}$, ocorre um aumento considerável, porém não tão rápido como na faixa $5-25^{\circ} \mathrm{C}$. Tal resultado é atribuído ao fato de que o $\mathrm{O}_{2}$ não se difunde com eficiência nessa temperatura.

Foi verificado que as inflorescências de Oncidium varicosum 'Samurai' que apresentaram maiores reservas de carboidratos, no final do armazenamento, obtiveram maior longevidade floral a $5^{\circ} \mathrm{C}$ (18 dias), $10^{\circ} \mathrm{C}$ (14 dias) e $20^{\circ} \mathrm{C}$ (12 dias). A longevidade depende da atividade respiratória, pois a

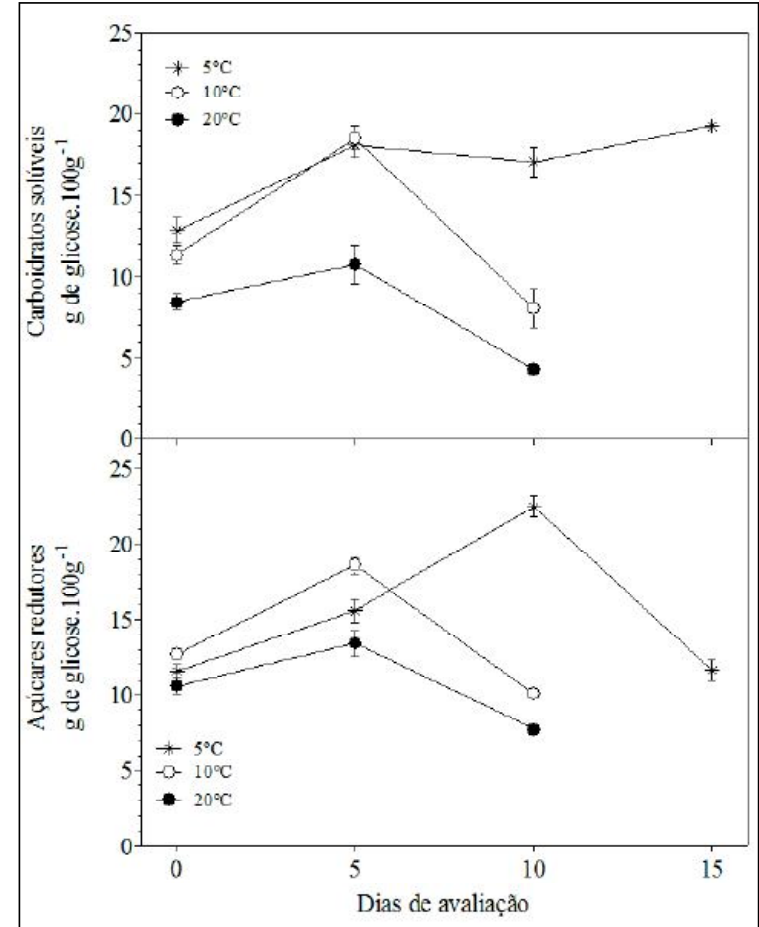

Figura 4 - Conteúdo de carboidratos solúveis e de açúcares redutores, em g de glicose $100 \mathrm{~g}^{-1}$, de inflorescências cortadas de Oncidium varicosum 'Samurai', armazenadas em diferentes temperaturas. Barras verticais representam o desvio padrão da média.

respiração está ligada ao crescimento e à senescência e constitui fonte de geração de calor. Além disso, quanto maior for a temperatura ao redor da flor maior será a taxa respiratória e o aumento de calor. As flores de Oncidium mantidas a 5 e $10^{\circ} \mathrm{C}$ mantiveram teores maiores de carboidratos, indicando que estes não foram usados durante o processo de senescência, o que resultou em vida útil mais longa.

\section{CONCLUSÃO}

O armazenamento de Oncidium em temperatura de $5^{\circ} \mathrm{C}$ favorece a manutenção do conteúdo relativo de água e de carboidratos solúveis, além da intensidade da cor, proporcionando maior longevidade às inflorescências.

\section{AGRADECIMENTO}

À Fundação de Amparo à Pesquisa do Estado de São Paulo (FAPESP), que nos concedeu recursos para a realização desta pesquisa (proc. n. 05/51186-0 e 05/60364-6). 


\section{REFERÊNCIAS}

CORREA, M.P. Dicionário das plantas úteis do Brasil e das exóticas cultivadas. Rio de Janeiro: Ministério da Agricultura, Instituto Brasileiro de Desenvolvimento Florestal, 1984. V.2

DEMMING-ADAMS, B. et al. Carotenoids 3: in vivo function of carotenoids in higher plants. Federation of American Society of Experimental Biology Journal, v.10, p.403412, 1996.

DUBOIS, M. et al. Colorimetric method for determination of sugars and related substances. Annals of Chemistry, v.28, n.3, p. 350-356, 1956.

HALEVY, A.H.; MAYAK, S. Senescence and postharvest physiology of cut flowers. Horticultural Reviews, v.3, p.59143, 1981 .

HENDRY, G.A.F.; PRICE, A.H. Stress indicators: chlorophylls and carotenoids. In: HENDRY, G.A.F.; GRIME, J.P. (Ed.). Methods in comparative plant ecology: a laboratory manual. London: Chapman \& Hall, 1993. p.148-152.

KAYS, S.J. Postharvest physiology of perishable plant products. New York: An Avi Book, 1991. 532p.

KERBAUY, G.B. Fisiologia vegetal. Rio de Janeiro: Guanabara Koogan, 2004. 452p.

KETSA, S.; THAMPITAKORN, F. Characteristics of ethylene production of Dendrobium orchid flowers. Acta Horticulturae, n.405, p.253-264, 1995.

KETSA, S. et al. Effect of precooling and ethylene absorbent on the quality of Dendrobium 'Pompadour' flowers. Acta Horticulturae, n.669, p.367-372, 2005.

KRAMER, P.J. Water relations of plants. New York: Academic, 1983. 489p.
MAROUSKY, F.J. Inhibition of vascular blockage and increased moisture retention in cut roses induced by $\mathrm{pH}, 8$ hidroxyquinoline citrate and sucrose. Journal of American Society for Horticultural Science, v.96, p.38-41, 1971.

MILLER, D. et al. Orquídeas do alto da serra da mata atlântica pluvial do sudeste do Brasil. Rio de Janeiro: Salamandra, 1996. 256p.

MINOLTA CORP. Precise color communication: color control from feeling to instrumentation. Ramsey: Minolta Corporation Instrument Systems Division, 1994. 49p.

NOWAK, J.; RUDNICKI, R.M. Postharvest handling and storage of cut flowers, florist greens and potted plants. Portland: Timber, 1990. 210p.

NOWAK, J. et al. Storage of cut flowers and ornamental plants: present status and future prospects. Postharvest News and Information, v.2, n.4, p.255-260, 1991.

PAULL, R.E.; CHANTRACHIT, T. Benzyladenine and the vase life of tropical ornamentals. Postharvest Biology and Technology, v.21, n.3, p.303-310, 2001.

ROBYT, J.F.; WHITE, B.J. Biochemical techniques: theory and practice. Illinois: Waveland, 1987. p.330-332.

ROGERS, M.N. An historical and critical review of postharvest physiology research on cut flowers. HortScience, v.8, n. 3, p.189-194, 1973.

SHAMAILA, M. et al. Sensory evaluation of strawberry fruit stored under modified atmosphere packing (MAP) by quantitative descriptive analysis. Journal Food Science, v.57, n.5, p.1168-1172, 1992.

WILLS, R.H.H. et al. Postharvest, and introduction to the physiology and handling of fruits and vegetables. Westport: AVI, 1998. 191p. 